\title{
Knowledge-Based QRS Detection Performed by a Cascade of Moving Average Filters
}

\author{
Lorenzo Bachi ${ }^{1}$, Lucia Billeci ${ }^{1}$, Maurizio Varanini ${ }^{1}$ \\ ${ }^{1}$ Institute of Clinical Physiology, National Research Council of Italy (IFC-CNR), Pisa, Italy
}

\begin{abstract}
The detection of QRS complexes is a crucial step since all the subsequent processing of the ECG signal is very sensitive to the accuracy of this detection.

This study presents an accurate and computationally efficient approach to heartbeat detection based on preprocessing and enhancement of the QRS complexes by means of cascades of moving averages.

Several derivative QRS-enhancing moving averages filters were defined which were characterized by different shapes of the impulsive response. In the initialization phase of the algorithm, the best filter for each record was selected by maximizing a specifically defined signal quality index. Detection of the QRS complex was based on a decision logic and a set of adaptive thresholds.

The MIT-BIH, QTDB and EU ST-T databases were considered for performance evaluation and comparison with the output of some publicly available QRS PanTompkins detectors, obtaining results comparable to the best reported in the literature ( $F 1=99.84 \%$ and $98.46 \%$ on MIT-BIH channel 1 and 2 respectively).
\end{abstract}

\section{Introduction}

Heartbeat detection is the prime concern in electrocardiography, as it serves as basis for more sophisticated estimation of the pathophysiological status of the heart, like heart rate variability (HRV) analysis. Heart rate estimation requires identification of the characteristic PQRST pattern taken by the electrical activity of the cardiac muscle, specifically its QRS components which correspond to ventricular depolarization. Electrocardiographic analysis constitutes the most common test to diagnose and monitor Cardiovascular Diseases and it is therefore mandatory for the heartbeat revelation process to be as accurate as possible. Skipping beats that have occurred could spring false alarms of cardiac pause, while overlooking potentially abnormal beats could lead to a wrong diagnosis.

QRS detection has been a topic of research for many decades, and with current technology an increasing number of different algorithmic approaches have become feasible [1]. The most well-known and referenced detection method is Pan and Tompkins' work [2], which achieved great results on both MIT-BIH [3] and AHA [4] databases and is still the landmark to which compare new solutions. Numerous different approaches have been proposed, with varying degree of complexity, such as wavelet transforms [5], support vector machines [6] and neural networks [7] to cite a few. While complex techniques offer greater signal characterization prospects, they are often computationally intensive, can be less resistant to noise and may not significantly improve accuracy in relation to more straightforward procedures. Besides, with the ever-increasing attention of the public towards wearable devices faster and numerically efficient processing is desirable. Elgendi [8] developed a fast QRS detector based on two moving averages of optimized length determined with a knowledge-based approach, designed for battery-driven devices.

The procedure proposed in this work derives from Taddei, Marchesi and Landucci's QRS detector [9]. Feature extraction is done through moving average filters, emphasizing execution speed while maintaining high accuracy on both normal and abnormal beats. Notably, ECG, as well as many biosignals, is defined by their evolution in time (PQRST waves), and cardiac events of pathophysiological interest are characterized by their waveform and by their occurrence. These time domain traits, which allow to discriminate different cardiac events, cannot be identified in frequency, with rare exceptions such as cardiac fibrillation. Therefore, we applied digital filtering for enhancing the QRS events detector by means of moving averages, avoiding frequency designed filters. In particular, a moving average cascade (MAC) was used to avoid the abrupt step of the moving average's rectangular time response.

For the sake of direct comparison between results, three different implementations of Pan - Tompkins algorithm have been implemented and run on the same databases.

\section{Methods}

2.1. Data 
The standard databases considered for development and evaluation of the algorithm are the MIT-BIH Arrhythmia, European ST-T and QT databases.

The MIT-BIH Arrhythmia database [3] is a collection of 48 two channel ambulatory ECG records, thirty minutes long each, selected from 4000 24-hour long ECG recordings sampled at $360 \mathrm{~Hz}$. Twenty-three of these records were chosen at random, the remaining twenty-five were handpicked to include not common clinically relevant arrhythmias.

The European ST-T database [10] consists of 90 two channel ambulatory ECG sampled at $250 \mathrm{~Hz}$, two hours long each, measured from 70 men and 8 women all diagnosed with myocardial ischemia. Excerpts were selected to include a variety of ECG abnormalities resulting from abnormal or pathological conditions.

The QT database [11] includes 105 two channel ECG recordings sampled at $250 \mathrm{~Hz}$, fifteen minutes long, chosen from other databases, with annotations on all characteristic waves.

\subsection{Algorithm description}

The QRS Detection algorithm included three steps: 1) reducing noise, i.e. non QRS signal changes; 2) enhancing QRS events; 3) decision logic on QRS event occurrence.

The first step was aimed to reduce the amplitude of slow waves like $\mathrm{T}$ and baseline movements and attenuating very short peaks due to noise. This task was fulfilled by the difference of two synchronous MACs of different impulsive response length. In particular a narrower MAC, whose impulsive response involved a $25 \mathrm{~ms}$ interval, and a wider MAC, whose impulsive response involved a $140 \mathrm{~ms}$ interval were used. The $25 \mathrm{~ms}$ length was chosen to balance the need to attenuate the noisy peaks and on the other hand the necessity of maintaining the amplitude of the QRS complexes' peaks, while the 140 ms length was selected as a compromise between the need to reduce slow movements and the need of not lowering the waves of the QRS complex.

Figure 1 shows the impulsive response of the resulting linear system (sampling frequency $=360 \mathrm{sps}$ ), which roughly corresponds to a band pass filter with cut-off frequencies at 7 and $17.6 \mathrm{~Hz}$.

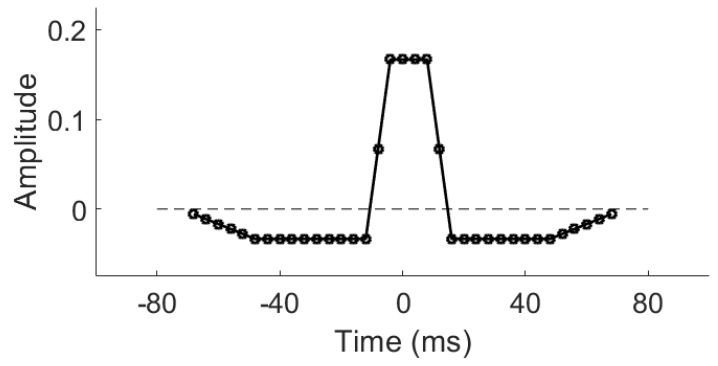

Figure 1. The impulsive responses of the difference of two synchronous MACs of different lengths.
The second processing step of the algorithm enhanced the QRS complex with respect to the other waves. This task was achieved by emphasizing the main characteristic of the QRS complex, that is, its high derivative which can involve an interval of approximately $100 \mathrm{~ms}$ length. Considering the mean duration of the rising (and falling) edge of the QRS waves, we designed a set of derivative systems consisting in the difference between two MACs shifted by $7 \div 11 \mathrm{~ms}$, each of them involving about $6 \div 8 \mathrm{~ms}$ of the signal.

The impulsive response of three derivative filters, as example, are shown in Figure 2.

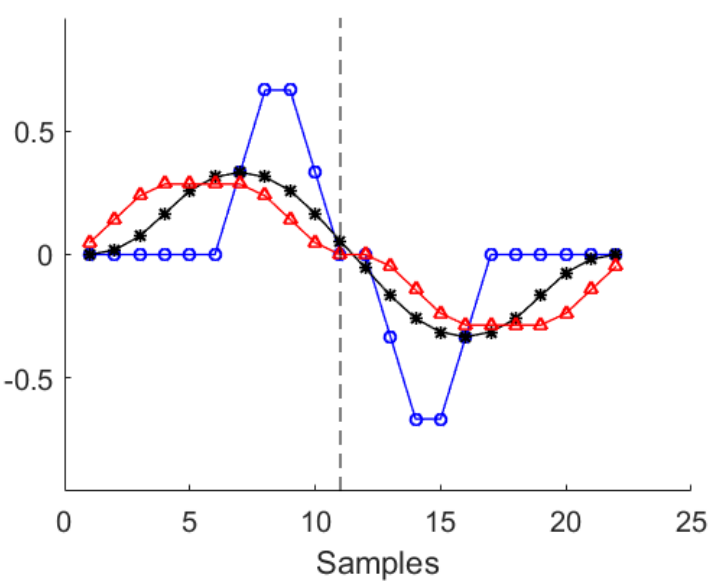

Figure 2. Examples of impulsive responses of three derivative systems, each one obtained as the difference between two delayed MACs.

During the initialization stage of the algorithm, occurring in the first 15 seconds of good quality ECG recording, the derivative filter which most enhances the QRS complexes was automatically chosen by calculating a signal quality index given by the following equation:

$$
\mathrm{SQI}=\frac{k_{s}+m D_{s}}{k_{n}+m D_{n}}
$$

where $m D_{s}$ is the trimmed mean of the maxima computed in successive windows of $1.6 \mathrm{~s}$, aimed at representing the QRS-specific high derivative values, and $m D_{n}$ is the trimmed mean of the maxima computed in successive windows of 0.09 seconds that accounts for the basal volume of noise. The terms $k_{s}$ and $k_{n}$ are small constants. Both $m D_{s}$ and $m D_{n}$ were computed for each of the predefined derivative system, and the one that maximizes $\mathrm{SQI}$ was selected as the derivative MAC filter, $\mathrm{MAC}_{2}[n]$, and applied to all the ECG record under analysis.

Finally, the third step of the QRS detection algorithm consisted in the comparison of the absolute value at the output of the derivative MAC filter with a threshold. Said threshold was first initialized and then updated in a 
bounded fashion with respect to its initial value, since spike artifacts and signal absence could divert its pertinence to the actual amplitude. The threshold $\mathrm{T}$ was defined as follows:

$$
T=\left\{\begin{array}{l}
\min \left(\bar{D}, 2.5 \cdot T^{(0)}\right) \\
\max \left(\bar{D}, 0.5 \cdot T^{(0)}\right)
\end{array}\right.
$$

where $\bar{D}$ was the average of the absolute derivative maximum, updated at every step and limited to 2.5 times the initial value of maximum T. Moreover, the value of the threshold used in the QRS detection decision rule decreased as the distance from the previous QRS detection increased. To ensure a consistent placement of the fiducial point of each detected QRS, the time instant of heartbeat occurrence was identified as the position of the maximum (or minimum) of the signed derivative signal. The decision between positive or negative derivative for fiducial point placement was performed in the initialization phase and then maintained throughout the rest of the recording.

\subsection{Performance evaluation}

The performance of the algorithm was evaluated in terms of the F1 score which is the harmonic mean of sensitivity (Se) and positive predictive value $(+\mathrm{P})$

$$
\begin{aligned}
& \mathrm{Se}=\frac{\mathrm{TP}}{\mathrm{TP}+\mathrm{FN}}+\mathrm{P}=\frac{\mathrm{TP}}{\mathrm{TP}+\mathrm{FP}} \\
& \mathrm{F} 1=\frac{2 \mathrm{TP}}{2 \mathrm{TP}+\mathrm{FN}+\mathrm{FP}}
\end{aligned}
$$

In addition we also calculated the sensitivity for atypical beats ( $\mathrm{Se}-\mathrm{A})$ as follows:

$$
\text { Se- } \mathrm{A}=\frac{\mathrm{TP}^{\mathrm{A}}}{\mathrm{TP}^{\mathrm{A}}+\mathrm{FN}^{\mathrm{A}}}
$$

where $\mathrm{TP}^{\mathrm{A}}$ and $\mathrm{FN}^{\mathrm{A}}$ are the confusion matrix measures considering only atypical beats.

Indeed, this metric is important to QRS detection, as atypical beats with distorted shapes are more difficult to catch and yet are often clinically relevant. The performance of the proposed algorithm was compared with the actual output of some publicly available detectors, based on the Pan-Tompkins architecture: Behar's [12], Wedekind's [13] and Sedghamiz's [14].

\section{Results}

Tables 1 and 2 show the results achieved by our algorithm and the best of the Pan-Tompkins implementations, which was Behar's one. We report gross and average F1 results for all records of the three considered databases in table 1, while we report Sens-A in table 2.

We also compared the computational load of the tested algorithms, showing that our moving average-based approach is three to four times faster than the fastest of the other tested programs: on average, the proposed algorithm was able to process an hour of ECG recording inn0.0898 seconds, while Behar's program took 0.3204 seconds. Execution time was measured on the same calculator (2013 notebook with I7-dual core-cpu@2.00 GHz, 6Gb RAM), with the same background conditions.

Table 1. F1 values of QRS detection on the considered databases for our work and the best Pan-Tompkins algorithm we tested.

\begin{tabular}{c|c|c|c}
\hline \multirow{2}{*}{ DB } & \multirow{2}{*}{ \#of beats } & \multicolumn{2}{|c}{ F1 \% } \\
\cline { 3 - 4 } & & This work & $\begin{array}{c}\text { Pan-Tmpk } \\
\text { (Behar) }\end{array}$ \\
\hline MIT ch1 & 109494 & 99.84 & 99.08 \\
MIT ch2 & 109494 & 98.46 & 96.98 \\
QTDB ch1 & 86995 & 99.94 & 99.95 \\
QTDB ch2 & 86995 & 99.93 & 98.64 \\
ST-T ch1 & 790565 & 99.70 & 99.48 \\
ST-T ch2 & 790565 & 99.52 & 98.21 \\
& & & \\
Gross & & 99.59 & 98.79 \\
Aver. & & 99.56 & 98.72 \\
\hline
\end{tabular}

Table 2. Sensitivity for atypical beats detection on the considered databases for our work and the best PanTompkins algorithm we tested.

\begin{tabular}{c|c|c|c}
\hline \multirow{2}{*}{ DB } & \multirow{2}{*}{$\begin{array}{c}\text { \# of } \\
\text { atypical } \\
\text { beats }\end{array}$} & \multicolumn{2}{|c}{ Se-A \% } \\
\cline { 3 - 4 } & & This work & $\begin{array}{c}\text { Pan- } \\
\text { Tompkins } \\
\text { (Behar) }\end{array}$ \\
\hline MIT ch1 & 34442 & 96.66 & 94.64 \\
MIT ch2 & 34442 & 94.95 & 94.67 \\
QTDB ch1 & 6288 & 98.47 & 98.16 \\
QTDB ch2 & 6288 & 98.12 & 91.48 \\
ST-T ch1 & 5927 & 98.21 & 97.74 \\
ST-T ch2 & 5927 & 94.57 & 94.43 \\
& & 96.21 & 94.86 \\
Gross & & 96.83 & 95.19 \\
Aver. & & \multicolumn{2}{|c}{}
\end{tabular}

\section{Discussion and conclusions}

In this paper, we presented a fast, accurate QRS detector where both the preprocessing and the derivative stages are based of combinations of moving average filters designed in the time domain, obtaining satisfying results comparable to the best ones reported in the literature [1]. 
Notably, the combinations of moving averages mentioned in this paper are equivalent to linear FIR filters, but contrary to FIR filters, they are designed in the time domain considering the differences in shape of the events to discriminate. This method avoids the presence of oscillatory artifacts that could be introduced by the standard frequency approach, while the oscillatory, sinclike frequency response introduced by the moving average of the signal is of little concern.

Performance evaluation was done on three popular, gold standard datasets: MIT-BIH, QTDB and EU ST-T, obtaining high results overall. Specifically, performance and execution time of the implemented algorithm were compared with three different implementations of the PanTompkins method. Our algorithm outperformed the others, reaching among all the considered Physionet databases a $99.59 \% \mathrm{~F} 1$ and $96.21 \% \mathrm{Se}-\mathrm{A}$. We observe that Se-A is lower, yet still satisfying (worst case scenario 94.75\%), possibly due to the heterogenous shapes of the atypical beats. Since in certain applications (as in dynamic ECG) atypical beats are of fundamental importance, we feel that this index is another major characteristic of any QRS detector besides accuracy, thus future work will be directed to improve this measure.

Importantly, our algorithm took on average 0.0898 seconds to process an hour of ECG recording, resulting to be three $\div$ four times faster than the quickest of the other tested programs. While the accuracy of the method is the fundamental performance metric, low execution time is nonetheless relevant for low-power devices such as wearables, which are increasingly popular in several applications. Therefore, we propose an algorithm that can be run on lighter hardware, like wearable sensors, without sacrificing reliability.

\section{Acknowledgments}

This work was funded from Cardioline S.p.A. and from the Italian MIUR through the PRIN project "Preclinical Tool for Advanced Translational Research with Ultrashort and Ultraintense X-ray Pulse" (prot. 20154F48P9).

\section{References}

[1] B.-U. Kohler, C. Hennig, and R. Orglmeister, "The Principles of Software QRS Detection," IEEE Eng. Med. Biol. Mag., vol. 21, no. 1, pp. 42-57, Jan. 2002, doi: 10.1109/51.993193.

[2] J. Pan and W. J. Tompkins, "A Real-Time QRS Detection Algorithm," IEEE Trans. Biomed. Eng., vol. BME-32, no. 3, pp. 230-236, Mar. 1985, doi: 10.1109/TBME.1985.325532.

[3] G. B. Moody and R. G. Mark, "The Impact of the MIT-BIH Arrhythmia Database," IEEE Eng. Med. Biol. Mag., vol. 20, no. 3, pp. 45-50, May 2001, doi: 10.1109/51.932724.

[4] "American Heart Association ECG Database USB," ECRI. https://www.ecri.org/american-heart-association-ecg- database-usb.

[5] Cuiwei Li, Chongxun Zheng, and Changfeng Tai, "Detection of ECG Characteristic Points Using Wavelet Transforms," IEEE Trans. Biomed. Eng., vol. 42, no. 1, pp. 21-28, Jan. 1995, doi: 10.1109/10.362922.

[6] S. S. Mehta and N. S. Lingayat, "Combined Entropy Based Method for Detection of QRS Complexes in 12-Lead Electrocardiogram Using SVM," Comput. Biol. Med., vol. 38, no. 1, pp. 138-145, Jan. 2008, doi: 10.1016/j.compbiomed.2007.08.003.

[7] Q. Xue, Y. H. Hu, and W. J. Tompkins, "Neural-NetworkBased Adaptive Matched Filtering for QRS Detection," IEEE Trans. Biomed. Eng., vol. 39, no. 4, pp. 317-329, Apr. 1992, doi: 10.1109/10.126604.

[8] M. Elgendi, "Fast QRS Detection with an Optimized Knowledge-Based Method: Evaluation on 11 Standard ECG Databases," PLOS ONE, vol. 8, no. 9, p. e73557, set 2013, doi: 10.1371/journal.pone.0073557.

[9] A. Taddei, C. Marchesi, and L. Landucci, "Performance Comparison of Fast QRS Detection Algorithms," in Ambulatory Monitoring: Cardiovascular system and allied applications Proceedings of a workshop held in Pisa, April 11-12, 1983. Sponsored by the Commission of the European Communities, as advised by the Committee on Medical and Public Health Research, C. Marchesi, Ed. Dordrecht: Springer Netherlands, 1984, pp. 189-207.

[10] A. Taddei, G. Distante, M. Emdin, P. Pisani, G. B. Moody, C. Zeelenberg, and C. Marchesi, "The European ST-T Database: Standard for Evaluating Systems for the Analysis of ST-T Changes in Ambulatory Electrocardiography," Eur. Heart J., vol. 13, no. 9, pp. 1164-1172, Sep. 1992, doi: 10.1093/oxfordjournals.eurheartj.a060332.

[11] P. Laguna, R. G. Mark, A. Goldberg, and G. B. Moody, “A Database for Evaluation of Algorithms for Measurement of QT and Other Waveform Intervals in the Ecg," in Computers in Cardiology 1997, Sep. 1997, pp. 673-676, doi: 10.1109/CIC.1997.648140.

[12] J. Behar, A. Johnson, G. D. Clifford, and J. Oster, "A Comparison of Single Channel Fetal ECG Extraction Methods," Ann. Biomed. Eng., vol. 42, no. 6, pp. 1340 1353, Jun. 2014, doi: 10.1007/s10439-014-0993-9.

[13] D. Wedekind, Danielwedekind/qrsdetector. 2020.

[14] H. Sedghamiz, Matlab Implementation of Pan Tompkins ECG QRS detector. 2014.

Address for correspondence:

Lorenzo Bachi

Institute of Clinical Physiology, National Research Council of Italy (IFC-CNR),

via Moruzzi 1, 56124, Pisa, Italy

bachi@ifc.cnr.it 